\title{
Integral and series representations of the digamma and polygamma functions
}

\author{
Mark W. Coffey \\ Department of Physics \\ Colorado School of Mines \\ Golden, CO 80401
}

(Received

2010)

August 10, 2010

\begin{abstract}
We obtain a variety of series and integral representations of the digamma function $\psi(a)$. These in turn provide representations of the evaluations $\psi(p / q)$ at rational argument and for the polygamma function $\psi^{(j)}$. The approach is through a limit definition of the zeroth Stieltjes constant $\gamma_{0}(a)=-\psi(a)$. Several other results are obtained, including product representations for $\exp \left[\gamma_{0}(a)\right]$ and for the Gamma function $\Gamma(a)$. In addition, we present series representations in terms of trigonometric integrals $\mathrm{Ci}$ and $\mathrm{Si}$ for $\psi(a)$ and the Euler constant $\gamma=-\psi(1)$.
\end{abstract}

\section{Key words and phrases}

Gamma function, digamma function, polygamma function, Euler constant, series representation, integral representation, Hurwitz zeta function, cosine integral, sine integral

2010 AMS codes

33B15, 33C20, 11Y60 


\section{Introduction and statement of results}

We recall the defining Laurent expansion of the Stieltjes constants $\gamma_{k}(a)[6$, 7, 5, 8, 20, 22,

$$
\zeta(s, a)=\frac{1}{s-1}+\sum_{n=0}^{\infty} \frac{(-1)^{n}}{n !} \gamma_{n}(a)(s-1)^{n},
$$

where $\zeta(s, a)$ is the Hurwitz zeta function. Notationally, we let $\zeta(s)=\zeta(s, 1)$ be the Riemann zeta function [11, 15, 17, 21], $\Gamma$ the Gamma function, $\psi=\Gamma^{\prime} / \Gamma$ be the digamma function (e.g., [1]) with $\gamma=-\psi(1)$ the Euler constant, $\psi^{(k)}$ be the polygamma functions [1], and ${ }_{p} F_{q}$ be the generalized hypergeometric function [2].

The Stieltjes constants may be expressed through the limit relation [3]

$$
\gamma_{n}(a)=\frac{(-1)^{n}}{n !} \lim _{N \rightarrow \infty}\left[\sum_{k=0}^{N} \frac{\ln ^{n}(k+a)}{k+a}-\frac{\ln ^{n+1}(N+a)}{n+1}\right], \quad n \geq 0 .
$$

Here, $a \notin\{0,-1,-2, \ldots\}$. For an asymptotic expression for these constants, even valid for moderate values of $n$, [16] (Section 2) may be consulted.

In this paper, we obtain various representations of the digamma function via the connection $\gamma_{0}(a)=-\psi(a)$ [22]. These in turn lead to many special cases, including the values $\psi(p / q)$ for rational argument, and further imply representations of the polygamma functions. We obtain product representations for $\exp \left[\gamma_{0}(a)\right]$ and $\Gamma(a)$. We present series representations for $\ln \Gamma(a), \psi(a)$, and $\gamma$ using the trigonometric integrals $\mathrm{Si}$ and $\mathrm{Ci}$. In addition, we provide several summations over parameterized values of $\mathrm{Ci}$.

The following is an example of representations that we develop. 
Proposition 1. Let Re $a>0$. Then we have

$$
\begin{aligned}
\gamma_{0}(a)=-\psi(a)=\frac{1}{2} \int_{0}^{\infty} \frac{1}{(t+a)(t+a+1)}{ }_{3} F_{2}(1,2,2 ; 3, t+a+2 ; 1) d t-\ln a \\
=-\ln a-\sum_{k=1}^{\infty} \frac{1}{k+1} \sum_{\ell=0}^{k}(-1)^{\ell}\left(\begin{array}{c}
k \\
\ell
\end{array}\right) \ln (\ell+a) \\
=-\ln a-\int_{0}^{1} u^{a-1}\left(\frac{1}{u-1}-\frac{1}{\ln u}\right) d u \\
=-\ln a-\int_{0}^{1} \int_{0}^{1} \frac{(x y)^{a-1}(1-x)}{(1-x y) \ln (x y)} d x d y \\
=-\ln a-\iint_{T} \frac{(1-Y)^{a-1}(1-X)}{X Y \ln (1-Y)} d X d Y \\
=-\ln a-\int_{0}^{\infty} \frac{1}{\sum_{k=2}^{\infty} \frac{1}{k^{2}} \frac{1}{\left(\begin{array}{c}
t+k+a-1 \\
k
\end{array}\right)} d t .}
\end{aligned}
$$

In (1.6b), $T$ is the triangle with vertices at $(1,0),(0,1)$, and $(1,1)$.

Thus from (1.4) we have

Corollary 1. We have for Re $a>0$

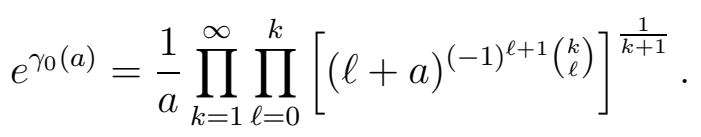

Proposition 1 and this Corollary subsume expressions for $\gamma$ given in [18].

In addition, from Proposition 1 follow representations for the polygamma functions, and these include

Corollary 2. We have for Re $a>0$

$$
\begin{aligned}
\psi^{(j)}(a)= & (-1)^{j-1} \frac{(j-1) !}{a^{j}}+(j-1) ! \sum_{k=1}^{\infty} \frac{1}{k+1} \sum_{\ell=0}^{k}(-1)^{\ell}\left(\begin{array}{c}
k \\
\ell
\end{array}\right) \frac{(-1)^{j-1}}{(\ell+a)^{j}} \\
& =(-1)^{j-1} \frac{(j-1) !}{a^{j}}+\int_{0}^{1} u^{a-1}\left(\frac{1}{u-1}-\frac{1}{\ln u}\right) \ln ^{j} u d u
\end{aligned}
$$




$$
\begin{gathered}
=(-1)^{j-1} \frac{(j-1) !}{a^{j}}+\int_{0}^{1} \int_{0}^{1} \frac{(x y)^{a-1}(1-x)}{(1-x y)} \ln ^{j-1}(x y) d x d y \\
=(-1)^{j-1} \frac{(j-1) !}{a^{j}}+\iint_{T} \frac{(1-Y)^{a-1}(1-X)}{X Y} \ln ^{j-1}(1-Y) d X d Y .
\end{gathered}
$$

Hence we obtain representations at positive integer arguments for harmonic numbers $H_{n} \equiv \sum_{k=1}^{n} 1 / k$ and generalized harmonic numbers $H_{n}^{(r)} \equiv \sum_{k=1}^{n} 1 / k^{r}$. For we have $H_{n}=\psi(n+1)-\psi(1)=\psi(n+1)+\gamma$ and

$$
H_{n}^{(r)}=\frac{(-1)^{r-1}}{(r-1) !}\left[\psi^{(r-1)}(n+1)-\psi^{(r-1)}(1)\right],
$$

where $\psi^{(r-1)}(1)=(-1)^{r}(r-1) ! \zeta(r)$.

Further, if we integrate $-\int_{1}^{a} \gamma_{0}(b) d b=\ln \Gamma(a)$ we have

Corollary 3. We have for Re $a>0$

$$
\begin{gathered}
\ln \Gamma(a)=a(\ln a-1)+1+\sum_{k=1}^{\infty} \frac{1}{k+1} \sum_{\ell=0}^{k}(-1)^{\ell}\left(\begin{array}{c}
k \\
\ell
\end{array}\right)[(\ell+a) \ln (\ell+a)-(\ell+1) \ln (\ell+1)] \\
=a(\ln a-1)+1+\int_{0}^{1}\left(u^{a-1}-1\right)\left(\frac{1}{u-1}-\frac{1}{\ln u}\right) \frac{d u}{\ln u} \\
=a(\ln a-1)-\frac{1}{2} \ln a+\frac{1}{2} \ln (2 \pi)+\int_{0}^{1} u^{a-1}\left(\frac{1}{u-1}-\frac{1}{\ln u}+\frac{1}{2}\right) \frac{d u}{\ln u} \\
=a(\ln a-1)+1+\int_{0}^{1} \int_{0}^{1} \frac{\left[(x y)^{a-1}-1\right](1-x)}{(1-x y) \ln ^{2}(x y)} d x d y \\
=a(\ln a-1)+1+\iint_{T} \frac{\left[1-(1-Y)^{a-1}\right](1-X)}{X Y \ln ^{2}(1-Y)} d X d Y .
\end{gathered}
$$

By inspection, we see that the right sides of (1.12)-(1.14) properly vanish at $a=1$. The term $a(\ln a-1)$ on the right sides there is the leading asymptotic form of $\ln \Gamma(a)$ when $a \rightarrow \infty$. In writing (1.13b), we have used (e.g., [19], p. 16)

$$
\int_{0}^{1}\left(\frac{1}{u-1}-\frac{1}{\ln u}+\frac{1}{2}\right) \frac{d u}{\ln u}=1-\frac{1}{2} \ln (2 \pi), \quad \int_{0}^{1} \frac{u^{a-1}-1}{\ln u} d u=\ln a, \quad \operatorname{Re} a>0,
$$


thereby recovering Binet's first expression for $\ln \Gamma(a)$.

Then we have from $(1.12)$

Corollary 4. We have for Re $a>0$

$$
\Gamma(a)=e^{1-a} a^{a} \prod_{k=1}^{\infty} \prod_{\ell=0}^{k}\left[\left[\frac{(\ell+a)^{\ell+a}}{(\ell+1)^{\ell+1}}\right]^{(-1)^{\ell}\left(\begin{array}{l}
k \\
\ell
\end{array}\right)}\right]^{\frac{1}{k+1}} .
$$

A multitude of summation formulas for the digamma function is known (e.g., 14], Section 55, [10]). Our integral representations in particular well provide a basis for further developing such summations. We simply give an example here. In light of the asymptotic relation as $a \rightarrow \infty$,

$$
\psi(a)=\ln a-\frac{1}{2 a}-\frac{1}{12 a^{2}}+O\left(\frac{1}{a^{4}}\right)
$$

we may consider the following.

Corollary 5. For $\operatorname{Re} \alpha>0$ and $\operatorname{Re}(\alpha+\beta)>0$, we have (a)

$$
\begin{gathered}
\sum_{n=1}^{\infty}\left[\psi(\alpha n+\beta)-\ln (\alpha n+\beta)+\frac{1}{2(\alpha n+\beta)}+\frac{1}{12(\alpha n+\beta)^{2}}\right] \\
=\int_{0}^{1} \frac{v^{\beta / \alpha}}{1-v}\left[\frac{1}{\alpha\left(v^{1 / \alpha}-1\right)}-\frac{1}{\ln v}+\frac{1}{2 \alpha}-\frac{1}{12 \alpha^{2}} \ln v\right] d v
\end{gathered}
$$

and (b) for $\alpha \gg 1$

$$
\sum_{n=1}^{\infty}\left[\psi(\alpha n+\beta)-\ln (\alpha n+\beta)+\frac{1}{2(\alpha n+\beta)}\right] \sim-\sum_{k=1}^{\infty} \frac{1}{\alpha^{2 k-1 / 2}} \frac{B_{2 k}}{2 k} \zeta\left(2 k, 1+\frac{\beta}{\alpha}\right)
$$

where $B_{j}$ is the $j$ th Bernoulli number.

Then we may determine the asymptotic dependence to all orders of a certain second moment of the Riemann xi function. For this, we put $\xi(s)=(s-1) \pi^{-s / 2} \Gamma(1+$ 
$s / 2) \zeta(s)$, and $\Xi(t) \equiv \xi(1 / 2+i t)$. We have

Corollary 6. We have as $\alpha \rightarrow \infty$

$$
\begin{gathered}
\int_{0}^{\infty}\left|\Xi\left(\frac{t}{2}\right) \Gamma\left(\frac{i t-1}{4}\right)\right|^{2} \frac{\cos [(t / 2) \ln \alpha]}{t^{2}+1} d t \\
\sim \frac{\pi^{3 / 2}}{2 \sqrt{\alpha}}[\ln \alpha+\ln (2 \pi)-\gamma]+\pi^{3 / 2} \sum_{k=1}^{\infty} \frac{1}{\alpha^{2 k-1 / 2}} \frac{B_{2 k}}{2 k} \zeta(2 k) .
\end{gathered}
$$

The moment integral here, going back to Ramanujan, is of interest from many points of view [4].

Let

$$
\operatorname{Ci}(z) \equiv-\int_{z}^{\infty} \frac{\cos t}{t} d t, \quad \operatorname{Si}(z) \equiv \int_{0}^{z} \frac{\sin t}{t} d t
$$

Then we have

Proposition 2. We have

$$
\gamma=\frac{1}{2}+\operatorname{Ci}(\pi)+\frac{1}{2 \pi}\left\{\sum_{j=1}^{\infty} \frac{1}{j+1}[\pi-2 \operatorname{Si}(\pi j)]+\sum_{j=2}^{\infty} \frac{1}{j-1}[\pi-2 \operatorname{Si}(\pi j)]\right\} .
$$

In this expression, $\mathrm{Ci}(\pi) \simeq 0.07366079$ and therefore the sum terms provide small corrections.

Proposition 3. We have for Re $a>0$

$$
\psi(a)=\ln a-\frac{1}{2 a}+\sum_{j=1}^{\infty}[2 \cos (2 \pi j a) \operatorname{Ci}(2 \pi j a)-\sin (2 \pi j a)[\pi-2 \operatorname{Si}(2 \pi j a)]]
$$

Then from $\psi(n+1)=\psi(n)+1 / n$ we have

Corollary 7. We have

$$
H_{n}=\ln n+\gamma+\frac{1}{2 n}+2 \sum_{j=1}^{\infty} \mathrm{Ci}(2 \pi n j)
$$


Alternatively, this result follows from the Euler-Maclaurin summation expression

$$
H_{n}=\ln n+\gamma+\frac{1}{2 n}+\int_{n}^{\infty} \frac{P_{1}(x)}{x^{2}} d x=\ln n+\gamma+\frac{1}{2 n}+\frac{1}{n} \int_{1}^{\infty} \frac{P_{1}(n v)}{v^{2}} d v,
$$

where $P_{1}$ is the polynomial given in (2.29). Similarly, for $r>1$ we have

$$
H_{n}^{(r)}=\zeta(r)+\frac{1}{2 n^{r}}-\frac{n^{1-r}}{r-1}+r \int_{n}^{\infty} \frac{P_{1}(x)}{x^{r+1}} d x=\zeta(r)+\frac{1}{2 n^{r}}-\frac{n^{1-r}}{r-1}+\frac{r}{n^{r}} \int_{1}^{\infty} \frac{P_{1}(n v)}{v^{r+1}} d v .
$$

Let $B_{n}(x)$ be the Bernoulli polynomial of degree $n, B_{n}=B_{n}(0)$ the $n$th Bernoulli number, and $B_{n}(1 / 2)=\left(2^{1-n}-1\right) B_{n}$ [1] (p. 805). Then we have the following. Proposition 4. Let $0<\beta \leq 2 \pi$. Then we have (a)

$$
\begin{gathered}
\sum_{n=1}^{\infty} \frac{\mathrm{Ci}(\beta n)}{n^{2}}=(\gamma+\ln \beta) \zeta(2)-\zeta^{\prime}(2)-\frac{\pi}{2} \beta+\frac{\beta^{2}}{8}, \\
\sum_{n=1}^{\infty}(-1)^{n} \frac{\mathrm{Ci}(\beta n)}{n^{2}}=\frac{1}{2}(\ln 2-\gamma-\ln \beta) \zeta(2)+\frac{1}{2} \zeta^{\prime}(2)+\frac{\beta^{2}}{8},
\end{gathered}
$$

(b)

$$
\begin{gathered}
\sum_{n=1}^{\infty} \frac{\mathrm{Ci}(\beta n)}{n^{4}}=(\gamma+\ln \beta) \zeta(4)-\zeta^{\prime}(4)+\frac{\beta^{2}}{12}\left(-\frac{\beta^{2}}{16}+\frac{\pi}{3} \beta-\frac{\pi^{2}}{2}\right), \\
\sum_{n=1}^{\infty}(-1)^{n} \frac{\mathrm{Ci}(\beta n)}{n^{4}}=(\ln 2-7 \gamma-7 \ln \beta) \frac{\zeta(4)}{8}+\frac{7}{8} \zeta^{\prime}(4)-\frac{\beta^{4}}{192}+\frac{\zeta(2)}{8} \beta^{2},
\end{gathered}
$$

(c) for integers $k \geq 1$

$$
\begin{gathered}
\sum_{n=1}^{\infty} \frac{\operatorname{Ci}(\beta n)}{n^{2 k}}=(\gamma+\ln \beta) \zeta(2 k)-\zeta^{\prime}(2 k)+\frac{(-1)^{k-1}}{2(2 k) !}(2 \pi)^{2 k} \int_{0}^{1} \frac{1}{v}\left[B_{2 k}\left(\frac{\beta v}{2 \pi}\right)-B_{2 k}\right] d v \\
\sum_{n=1}^{\infty}(-1)^{n} \frac{\operatorname{Ci}(\beta n)}{n^{2 k}}=(\gamma+\ln \beta)\left(2^{1-2 k}-1\right) \zeta(2 k)+\left(1-2^{1-2 k}\right) \zeta^{\prime}(2 k)+2^{1-2 k}(\ln 2) \zeta(2 k) \\
+\frac{(-1)^{k-1}}{2(2 k) !}(2 \pi)^{2 k} \int_{0}^{1} \frac{1}{v}\left[B_{2 k}\left(\frac{\beta v+\pi}{2 \pi}\right)-B_{2 k}\left(\frac{1}{2}\right)\right] d v,
\end{gathered}
$$


(d) for $\operatorname{Re} a>1$,

$$
\begin{aligned}
& \sum_{n=1}^{\infty} \frac{\operatorname{Ci}(\beta n)}{n^{a}}=(\gamma+\ln \beta) \zeta(a)-\zeta^{\prime}(a)+\int_{0}^{1}\left\{\frac{(2 \pi)^{a}}{4 \Gamma(a)} \sec \left(\frac{\pi a}{2}\right)\left[\zeta\left(1-a, 1-\frac{\beta v}{2 \pi}\right)+\zeta\left(1-a, \frac{\beta v}{2 \pi}\right)\right]\right. \\
& -\zeta(a)\} \frac{d v}{v} \\
& \sum_{n=1}^{\infty}(-1)^{n} \frac{\mathrm{Ci}(\beta n)}{n^{a}}=(\gamma+\ln \beta)\left(2^{1-a}-1\right) \zeta(a)+\left(1-2^{1-a}\right) \zeta^{\prime}(a)+2^{1-a} \zeta(a) \ln 2 \\
& +\int_{0}^{1}\left\{\frac{(2 \pi)^{a}}{4 \Gamma(a)} \sec \left(\frac{\pi a}{2}\right)\left[\zeta\left(1-a, \frac{\pi-\beta v}{2 \pi}\right)+\zeta\left(1-a, \frac{\pi+\beta v}{2 \pi}\right)\right]+\left(1-2^{1-a}\right) \zeta(a)\right\} \frac{d v}{v},
\end{aligned}
$$

(e) for $\operatorname{Re} a>1$,

$$
\begin{gathered}
\sum_{n=1}^{\infty} \frac{1}{(2 n+1)^{a}} \operatorname{Ci}(\beta n)=(\gamma+\ln \beta)\left[\left(1-2^{-a}\right) \zeta(a)-1\right]+S(a) \\
+\int_{0}^{1}\left\{\frac { ( 2 \pi ) ^ { a } } { 4 \Gamma ( a ) } \operatorname { c s c } ( \pi a ) \left[-\sin \left(\frac{\beta v+\pi a}{2}\right)\left[\zeta\left(1-a, \frac{\beta v+2 \pi}{4 \pi}\right)-\zeta\left(1-a, \frac{\beta v}{4 \pi}\right)\right]\right.\right. \\
\left.\left.+\sin \left(\frac{\beta v-\pi a}{2}\right)\left[\zeta\left(1-a, \frac{2 \pi-\beta v}{4 \pi}\right)-\zeta\left(1-a, 1-\frac{\beta v}{4 \pi}\right)\right]\right]+\left(2^{-a}-1\right) \zeta(a)\right\} \frac{d v}{v} \\
+\sum_{0=1}^{1} \frac{(-1)^{n}}{(2 n+1)^{a}} \operatorname{Ci}(\beta n)=(\gamma+\ln \beta)\left[4^{-a}(\zeta(a, 1 / 4)-\zeta(a, 3 / 4))-1\right]+T(a) \\
+\cos \left(\frac { \beta v - \pi a } { 4 \Gamma ( a ) } \operatorname { c s c } ( \pi a ) \left[\cos \left(\frac{\beta v+\pi a}{2}\right)\left[\zeta\left(1-a, \frac{\beta v+\pi}{4 \pi}\right)-\zeta\left(1-a, \frac{\beta v+3 \pi}{4 \pi}\right)\right]\right.\right.
\end{gathered}
$$

where,

$$
S(a) \equiv \sum_{n=2}^{\infty} \frac{\ln n}{(2 n+1)^{a}}, \quad T(a) \equiv \sum_{n=2}^{\infty} \frac{(-1)^{n} \ln n}{(2 n+1)^{a}}
$$


and (f) for $|z| \leq 1$,

$$
\begin{gathered}
\sum_{n=1}^{\infty} \frac{z^{n}}{n(n+1)} \operatorname{Ci}(\beta n)=(\gamma+\ln \beta)\left[1-\ln (1-z)+\frac{1}{z} \ln (1-z)\right]+\sum_{n=1}^{\infty} \frac{z^{n} \ln n}{n(n+1)} \\
\left.+\frac{1}{z} \int_{0}^{1}[(z-1) \ln (1-z)]-\frac{1}{2}(z-\cos \beta v) \ln \left(1-2 z \cos \beta v+z^{2}\right)-\sin \beta v \tan ^{-1}\left(\frac{z \sin \beta v}{1-z \cos \beta}\right)\right] \frac{d v}{v} .
\end{gathered}
$$

\section{Proof of Propositions}

We let $\psi^{\prime}$ be the trigamma function and $(b)_{n}=\Gamma(b+n) / \Gamma(b)$ be the Pochhammer symbol.

Proposition 1. Preliminary relations are contained in

Lemma 1. We have

$$
\begin{gathered}
\gamma_{0}(a)=\sum_{k=0}^{\infty} \int_{0}^{\infty}\left[\frac{1}{(t+k+a)^{2}}-\frac{1}{(t+k+a+1)(t+k+a)}\right] d t-\ln a \\
=\sum_{k=0}^{\infty} \int_{0}^{\infty} \frac{1}{(t+k+a)^{2}} \frac{d t}{(t+k+a+1)}-\ln a \\
=\int_{0}^{\infty}\left[\psi^{\prime}(t+a)-\frac{1}{t+a}\right] d t-\ln a=-\psi(a) .
\end{gathered}
$$

Proof. From relation (1.2) we have

$$
\begin{gathered}
\gamma_{0}(a)=\lim _{N \rightarrow \infty} \sum_{k=0}^{N}\left[\frac{1}{k+a}-\ln (N+a)\right] \\
=\lim _{N \rightarrow \infty} \sum_{k=0}^{N}\left[\frac{1}{k+a}-\ln \left(\frac{k+a+1}{k+a}\right)\right]-\ln a \\
=\sum_{k=0}^{\infty} \int_{0}^{\infty}\left[\frac{1}{(t+k+a)^{2}}-\frac{1}{(t+k+a+1)(t+k+a)}\right] d t-\ln a .
\end{gathered}
$$


The rest of the Lemma follows easily upon noting (e.g., p. 259 of [1])

$$
\psi^{\prime}(z)=\zeta(2, z)=\sum_{n=0}^{\infty} \frac{1}{(n+z)^{2}}
$$

We now write from the Lemma

$$
\gamma_{0}(a)=\int_{0}^{\infty} \frac{1}{(t+a)^{2}} \frac{1}{(t+a+1)} \sum_{k=0}^{\infty} \frac{(t+a)^{2}(t+a+1)}{(t+k+a)^{2}(t+k+a+1)} d t-\ln a .
$$

The integrand of (2.2) being absolutely convergent, the interchange of summation and integration is justified. In order to achieve hypergeometric form, we note the ratios

$$
\frac{(t+a)_{k}}{(t+a+1)_{k}}=\frac{t+a}{t+a+k}, \quad \frac{(t+a)_{k}}{(t+a+2)_{k}}=\frac{(t+a)(t+a+1)}{(t+a+k)(t+a+k+1)} .
$$

Upon using the series definition of the function ${ }_{3} F_{2}$, we therefore obtain

$$
\begin{gathered}
\gamma_{0}(a)=\int_{0}^{\infty} \frac{1}{(t+a)^{2}} \frac{1}{(t+a+1)}{ }_{3} F_{2}(1, t+a, t+a ; t+a+1, t+a+2 ; 1) d t-\ln a \\
=\frac{1}{2} \int_{0}^{\infty} \frac{1}{(t+a)^{2}} \frac{1}{(t+a+1)}{ }_{3} F_{2}(1,2,2 ; 3, t+a+2 ; 1) d t-\ln a .
\end{gathered}
$$

Here, we have used the transformation [18] (6), valid for $\operatorname{Re} s>0$ and $\operatorname{Re} v-t>0$,

$$
{ }_{3} F_{2}(1, s, t ; s+1, v ; 1)=\frac{s}{v-t}{ }_{3} F_{2}(1, v-t, v-s ; v-t+1, v ; 1) .
$$

We have obtained (1.3).

Next we have

$$
\begin{gathered}
{ }_{3} F_{2}(1,2,2 ; 3, t+a+2 ; 1)=\sum_{j=0}^{\infty} \frac{(2)_{j}^{2}}{(3)_{j}(t+a+2)_{j}}=2 \sum_{j=0}^{\infty} \frac{(j+1) !}{(j+2)(t+a+2)_{j}} \\
=2 \sum_{j=1}^{\infty} \frac{1}{j+1} \frac{j !}{(t+a+2)_{j-1}}=2 \sum_{j=1}^{\infty} \frac{1}{j+1} \frac{j !(t+a+j+1)}{(t+a+2)_{j}} .
\end{gathered}
$$


The integral in (2.6) becomes

$\frac{1}{2} \int_{0}^{\infty} \frac{{ }_{3} F_{2}(1,2,2 ; 3, t+a+2 ; 1)}{(t+a)^{2}} \frac{d t}{(t+a+1)}=\int_{0}^{\infty} \sum_{j=1}^{\infty} \frac{j !}{(j+1)} \frac{(t+a+j+1) d t}{(t+a)(t+a+1)(t+a+2)_{j}}$.

Therefore, from (2.6) we find

$$
\gamma_{0}(a)=-\ln a+\int_{0}^{\infty} \sum_{k=1}^{\infty} \frac{k !}{(k+1)(t+a)(t+a+1) \cdots(t+k+a)} d t
$$

We can carry out the integration by using the partial fraction decomposition [13

$$
\frac{N !}{x(x+1) \cdots(x+N)}=\sum_{k=0}^{N}\left(\begin{array}{l}
N \\
k
\end{array}\right) \frac{(-1)^{k}}{(x+k)} \text {. }
$$

We then have (1.4).

If we employ the Beta function integral

$$
\int_{0}^{1} u^{t+a-1}(1-u)^{k} d u=B(t+a, k+1)=\frac{k !}{(t+a)(t+a+1)_{k}}
$$

in (2.10) we find

$$
\begin{gathered}
\gamma_{0}(a)=-\ln a+\int_{0}^{\infty} \sum_{k=1}^{\infty} \int_{0}^{1} \frac{u^{t+a-1}(1-u)^{k}}{k+1} d u d t \\
=-\ln a+\int_{0}^{\infty} \int_{0}^{1} u^{t+a-1}\left(\frac{\ln u}{u-1}-1\right) d u d t .
\end{gathered}
$$

Performing the integral over $t$ gives (1.5).

We may rewrite (2.13) as

$$
\begin{aligned}
\gamma_{0}(a) & =-\ln a+\int_{0}^{\infty} \int_{0}^{1} \int_{0}^{1-u} \frac{u^{t+a-1}}{(1-u)} \frac{v}{(1-v)} d v d u d t \\
& =-\ln a+\int_{0}^{1} \int_{0}^{1-u} \frac{u^{a-1}}{(u-1)} \frac{v}{(1-v)} \frac{d v d u}{\ln u} .
\end{aligned}
$$


We now put $u=x y, v=1-x$ and use the Jacobian $\partial(u, v) / \partial(x, y)=x$ to obtain (1.6a).

Putting $x=X$ and $y=(1-Y) / X$, with Jacobian of transformation $\partial(X, Y) / \partial(x, y)=$ $-x$, in (1.6a) yields (1.6b). Using $-1 / \ln (1-Y)=\int_{0}^{\infty}(1-Y)^{t} d t$ in $(1.6 \mathrm{~b})$ gives

$$
\begin{gathered}
\gamma_{0}(a)=-\psi(a)=-\ln a+\iint_{T} \int_{0}^{\infty} \frac{(1-Y)^{t+a-1}}{X Y}(1-X) d t d X d Y \\
=-\ln a+\int_{0}^{\infty} \int_{0}^{1} \int_{1-Y}^{1} \frac{(1-X)}{X Y}(1-Y)^{t+a-1} d X d Y d t \\
=-\ln a-\int_{0}^{\infty} \int_{0}^{1} \frac{(1-Y)^{t+a-1}}{Y}[Y+\ln (1-Y)] d Y d t \\
=-\ln a-\int_{0}^{\infty} \sum_{k=2}^{\infty} \frac{1}{k} \int_{0}^{1}(1-Y)^{t+a-1} Y^{k-1} d Y d t \\
=-\ln a-\int_{0}^{\infty} \sum_{k=2}^{\infty} \frac{1}{k} B(k, t+a) d t
\end{gathered}
$$

where we employed the Beta function integral of (2.12). Then we have the expressions

$$
\begin{aligned}
\gamma_{0}(a) & =-\ln a-\int_{0}^{\infty} \sum_{k=2}^{\infty} \frac{1}{k} \frac{\Gamma(k) \Gamma(t+a)}{\Gamma(t+k+a)} d t \\
& =-\ln a-\int_{0}^{\infty} \sum_{k=2}^{\infty} \frac{\Gamma(k)}{k} \frac{d t}{(t+a)_{k}},
\end{aligned}
$$

that are equivalent to $(1.6 \mathrm{c})$.

Corollary 5. From (1.5) we have

$$
\psi(a)=\ln a-\frac{1}{2 a}-\frac{1}{12 a^{2}}+\int_{0}^{1} u^{a-1}\left(\frac{1}{u-1}-\frac{1}{\ln u}+\frac{1}{2}-\frac{1}{12} \ln u\right) d u
$$

We then interchange summation and integration to find

$$
\sum_{n=1}^{\infty}\left[\psi(\alpha n+\beta)-\ln (\alpha n+\beta)+\frac{1}{2(\alpha n+\beta)}+\frac{1}{12(\alpha n+\beta)^{2}}\right]
$$




$$
=\int_{0}^{1} \frac{u^{\alpha+\beta-1}}{1-u^{\alpha}}\left[\frac{1}{u-1}-\frac{1}{\ln u}+\frac{1}{2}-\frac{1}{12} \ln u\right] d u .
$$

Making the change of variable $v=u^{\alpha}$ gives part (a) of the Corollary. For part (b), we use the asymptotic relation for the digamma function as $z \rightarrow \infty$

$$
\psi(z)=\ln z-\frac{1}{2 z}-\sum_{k=1}^{\infty} \frac{B_{2 k}}{2 k z^{2 k}} .
$$

Corollary 6 . This follows from the $\beta=0$ case of Corollary $5(\mathrm{~b})$, together with the reciprocity relation with $\alpha, \beta>0$ and $\alpha \beta=1$ [4]

$$
\begin{gathered}
\sqrt{\alpha}\left[\frac{\gamma-\ln (2 \pi \alpha)}{2 \alpha}+\sum_{n=1}^{\infty} \phi(n \alpha)\right]=\sqrt{\beta}\left[\frac{\gamma-\ln (2 \pi \beta)}{2 \beta}+\sum_{n=1}^{\infty} \phi(n \beta)\right] \\
=-\frac{1}{\pi^{3 / 2}} \int_{0}^{\infty}\left|\Xi\left(\frac{t}{2}\right) \Gamma\left(\frac{i t-1}{4}\right)\right|^{2} \frac{\cos [(t / 2) \ln \alpha]}{t^{2}+1} d t
\end{gathered}
$$

where $\phi(x)=\psi(x)+1 / 2 x-\ln x$.

An alternative approach is to expand the integrand of Corollary 5(a), using

$$
v^{1 / \alpha}-1=\sum_{j=1}^{\infty} \frac{\ln ^{j} v}{j ! \alpha^{j}}
$$

followed by the use of the integrals $\int_{0}^{1}\left[\ln ^{j} v /(1-v)\right] d v=(-1)^{j} j ! \zeta(j+1)$.

We note that the reciprocity relation itself provides the complementary asymptotic relation as $\alpha \rightarrow 0$. For then $\beta=1 / \alpha \rightarrow \infty$.

We also note that the leading term of the asymptotic relation in Corollary 6 is connected with the skew self-reciprocal inverse Fourier cosine transform

$$
\mathcal{F}_{c}^{-1}\left(\frac{\ln \alpha}{\sqrt{\alpha}}\right)=-\frac{1}{\sqrt{t}}\left(\ln t+\gamma+\frac{\pi}{2}+2 \ln 2\right) .
$$


This transform may be calculated by logarithmic differentiation with respect to $x$ of the integral $\sqrt{\frac{2}{\pi}} \int_{0}^{\infty} \alpha^{x} \cos (\alpha t) d \alpha$ with $-1<\operatorname{Re} x<0$, at $x=-1 / 2$.

Remarks. If in (1.5) we put $u=\exp (-t)$, we have

$$
\gamma_{0}(a)=-\ln a+\int_{0}^{\infty} e^{-a t}\left(\frac{1}{1-e^{-t}}-\frac{1}{t}\right) d t
$$

thereby recovering formula 8.361 .8 of [12] (p. 943) for the digamma function. This formula is also recovered if we use an integral representation for $\ln , \ln z=\int_{0}^{\infty}\left(e^{-t}-\right.$ $\left.e^{-z t}\right)(d t / t)$ for $\operatorname{Re} z>0$, in (1.4). For then we have

$$
\begin{aligned}
\gamma_{0}(a) & =-\ln a+\sum_{k=1}^{\infty} \frac{1}{k+1} \int_{0}^{\infty} \frac{e^{-a t}}{t}\left(1-e^{-t}\right)^{k} d t \\
& =-\ln a-\int_{0}^{\infty} e^{-a t}\left(\frac{1}{t}-\frac{1}{1-e^{-t}}\right) d t .
\end{aligned}
$$

If in $(2.15 \mathrm{~b})$ we instead carry out the integration over $Y$, we recover the integral of (2.1).

Special values of $\gamma_{0}$ are $\gamma_{0}(1 / 2)=\gamma+2 \ln 2$, and

$$
\gamma_{0}\left(n+\frac{1}{2}\right)=\gamma+2 \ln 2-2 \sum_{k=0}^{n-1} \frac{1}{2 k+1} .
$$

Further, $\gamma_{0}(1 / 4)=\gamma+\pi / 2+3 \ln 2$, and $\gamma(3 / 4)=\gamma-\pi / 2+3 \ln 2$, giving for instance $\gamma_{0}(1 / 4)-\gamma_{0}(3 / 4)=\pi$. More generally, for rational arguments we have the following ([2], p. 13 or [19], p 19).

\section{Theorem.}

$$
\psi\left(\frac{p}{q}\right)=-\gamma-\frac{\pi}{2} \cot \frac{\pi p}{q}-\ln q+2 \sum_{n=1}^{[q / 2]} ' \cos \frac{2 \pi n p}{q} \ln \left(2 \sin \frac{\pi n}{q}\right),
$$


where $0<p<q$; $\sum^{\prime}$ means that when $q$ is even the term with index $n=q / 2$ is divided by 2 .

Therefore, we have found representations for all the values of (2.21).

The digamma and polygamma functions satisfy many properties including functional equations, duplication and multiplication formulas, and reflection formulas. All such properties must be inherent in our various series and integral representations. As an illustration, we have

Corollary 8 (multiplication formula). For integers $m \geq 1$ we have

$$
\gamma_{0}(m a)=-\ln m+\frac{1}{m} \sum_{k=0}^{m-1} \gamma_{0}\left(a+\frac{k}{m}\right) .
$$

Proof. From (1.5) we have

$$
\begin{aligned}
& \gamma_{0}(m a)=-\ln m-\ln a-\int_{0}^{1} u^{m a-1}\left(\frac{1}{u-1}-\frac{1}{\ln u}\right) d u \\
& =-\ln m-\ln a-\int_{0}^{1} v^{a-1}\left[\frac{1}{m\left(v^{1 / m}-1\right)}-\frac{1}{\ln v}\right] d v .
\end{aligned}
$$

The Corollary then follows from the factorization

$$
\frac{1}{v^{1 / m}-1}=\frac{\sum_{k=0}^{m-1} v^{k / m}}{v-1}
$$

Proposition 2. We have previously obtained the integral representation [7] (2.86)

$$
\gamma=\int_{-\infty}^{\infty} \frac{e^{z} \ln \left(1+e^{-z}\right)}{z^{2}+\pi^{2}} d z
$$

The idea of the proof is to suitably expand the logarithm of the integrand, and then to perform termwise integration. For this we write

$$
\gamma=\left(\int_{0}^{\infty}+\int_{-\infty}^{0}\right) \frac{e^{z} \ln \left(1+e^{-z}\right)}{z^{2}+\pi^{2}} d z
$$




$$
\begin{gathered}
=\sum_{j=1}^{\infty} \frac{(-1)^{j-1}}{j} \int_{0}^{\infty} \frac{e^{-(j-1) z}}{z^{2}+\pi^{2}} d z+\int_{0}^{\infty} e^{-y} \frac{\left[y+\ln \left(1+e^{-y}\right)\right]}{y^{2}+\pi^{2}} d y \\
=\frac{1}{2}+\operatorname{Ci}(\pi)+\frac{1}{2 \pi} \sum_{j=2}^{\infty} \frac{1}{j}(\pi+2 \operatorname{Si}[\pi(1-j)])+\sum_{j=1}^{\infty} \frac{(-1)^{j-1}}{j} \int_{0}^{\infty} \frac{e^{-(j+1) y}}{y^{2}+\pi^{2}} d y \\
=\frac{1}{2}+\operatorname{Ci}(\pi)+\frac{1}{2 \pi} \sum_{j=2}^{\infty} \frac{1}{j}(\pi-2 \operatorname{Si}[\pi(j-1)])+\frac{1}{2 \pi} \sum_{j=1}^{\infty} \frac{1}{j}(\pi-2 \operatorname{Si}[\pi(j+1)]) .
\end{gathered}
$$

Shifting the index in the summations gives the expression (1.20).

Remarks. The asymptotic forms of $\mathrm{Si}$ and $\mathrm{Ci}$ are easily obtained to any order by repeated integration by parts. It is then easy to see that the summations in $(1.20)$ have summands that are $O\left(1 / j^{2}\right)$, and additionally these leading terms have sign alternation according to $(-1)^{j}$.

For comparison purposes, we recall an earlier result [9]

$$
1-\gamma=\frac{1}{2}+2 \sum_{j=1}^{\infty} \mathrm{Ci}(2 \pi j)
$$

that readily shows how to develop $1-\gamma$ from $1 / 2$ with a series of corrections, and the leading terms in the corrections are easily written.

We recall that the constant (e.g., [19], p. 345)

$$
1-\gamma=\int_{1}^{\infty} \frac{\{t\}}{t^{2}} d t
$$

where the fractional part $\{t\}=t-[t]$. We have that $P_{1}(x)=B_{1}(x-[x])=x-[x]-1 / 2$, the first periodized Bernoulli polynomial, has the standard Fourier series [1] (p. 805),

$$
P_{1}(x)=-\sum_{j=1}^{\infty} \frac{\sin (2 \pi j x)}{\pi j} .
$$


Inserted into (2.22), we have

$$
\begin{aligned}
\int_{1}^{\infty} \frac{\{t\}}{t^{2}} d t & =\frac{1}{2}-\frac{1}{\pi} \sum_{j=1}^{\infty} \frac{1}{j} \int_{1}^{\infty} \sin (2 \pi j t) \frac{d t}{t^{2}} \\
& =\frac{1}{2}+2 \sum_{j=1}^{\infty} \operatorname{Ci}(2 \pi j),
\end{aligned}
$$

where we integrated by parts and made a simple change of variable.

As a second proof of (2.27) we have the following. We write

$$
\begin{gathered}
\sum_{j=1}^{\infty} \operatorname{Ci}(2 \pi j)=-\sum_{j=1}^{\infty} \int_{2 \pi j}^{\infty} \frac{\cos t}{t} d t=-\sum_{j=1}^{\infty} \int_{0}^{\infty} \frac{\cos (v+2 \pi j)}{v+2 \pi j} d v=-\sum_{j=1}^{\infty} \int_{0}^{\infty} \frac{\cos v}{v+2 \pi j} d v \\
=-\sum_{j=1}^{\infty} \int_{0}^{\infty} \cos v d v \int_{0}^{\infty} e^{-(v+2 \pi j) x} d x=-\int_{0}^{\infty} \cos v \int_{0}^{\infty} \frac{e^{-x v} d x}{e^{2 \pi x}-1} \\
=-\int_{0}^{\infty} \frac{x d x}{\left(x^{2}+1\right)\left(e^{2 \pi x}-1\right)}=\frac{1}{2}\left(\frac{1}{2}-\gamma\right)
\end{gathered}
$$

Here the interchange of summation and integration is justfied by the absolute convergence of the $x$-integration. In the last step, we applied Hermite's expression for the digamma function ([19], p. 91 or [12], 8.361.3, p. 943).

Further representations for combinations of the Euler constant and zeta values may be obtained from the following.

Lemma 2. We have for $k \geq 2$

$$
I_{k} \equiv \int_{1}^{\infty} \frac{\{t\}^{k}}{t^{k+1}} d t=1-\gamma-\sum_{j=2}^{k} \frac{[\zeta(j)-1]}{j} .
$$

We provide an operational proof, using the Dirac delta function $\delta$. We have, integrating by parts,

$$
I_{k}=\frac{1}{k}\left[\int_{1}^{\infty} \frac{1}{t^{k}}\left(\frac{d}{d t}\{t\}^{k}\right) d t-\left.\frac{\{t\}^{k}}{t^{k}}\right|_{1} ^{\infty}\right]
$$




$$
\begin{gathered}
=\int_{1}^{\infty} \frac{\{t\}^{k-1}}{t^{k}} d t-\int_{1}^{\infty} \frac{\{t\}^{k-1}}{t^{k}} d[t]+\frac{1}{k} \\
=I_{k-1}-\sum_{j=2}^{\infty} \frac{1}{j^{k}}+\frac{1}{k} .
\end{gathered}
$$

Herein, we used $d[t]=\sum_{j} \delta(t-j) d t$.

Of course $I_{k} \rightarrow 0$ as $k \rightarrow \infty$, and we have the simple bound $I_{k} \leq 1 / k$.

We let $B_{k}(x)$ be the Bernoulli polynomials and $P_{k}(x)=B_{k}(x-[x])$ their periodized form. In applying Lemma 2 we may use the relation

$$
\{x\}^{n}=(x-[x])^{n}=\frac{1}{n+1} \sum_{k=0}^{n}\left(\begin{array}{c}
n+1 \\
k
\end{array}\right) P_{k}(x),
$$

with $B_{0}(x)=1$, and the Fourier series for $n \geq 1$ [1] (p. 805)

$P_{2 n-1}(x)=(-1)^{n} \frac{2(2 n-1) !}{(2 \pi)^{2 n-1}} \sum_{j=1}^{\infty} \frac{\sin (2 \pi j x)}{j^{2 n-1}}, \quad P_{2 n}(x)=(-1)^{n-1} \frac{2(2 n) !}{(2 \pi)^{2 n}} \sum_{j=1}^{\infty} \frac{\cos (2 \pi j x)}{j^{2 n}}$.

Then we have

Corollary 9. We have (a)

$$
I_{2}=\frac{3}{2}-\gamma-\frac{1}{2} \zeta(2)=\frac{1}{4}+\sum_{j=1}^{\infty}\left[2 \mathrm{Ci}(2 \pi j)+j \pi^{2}-1-2 j \pi \mathrm{Si}(2 \pi j)\right]
$$

and (b)

$$
I_{3}=\frac{11}{6}-\gamma-\frac{1}{2} \zeta(2)-\frac{1}{3} \zeta(3)=\frac{1}{4}+\sum_{j=1}^{\infty}\left[2\left(1-\frac{2}{3} j^{2} \pi^{2}\right) \mathrm{Ci}(2 \pi j)-\frac{4}{3}+j \pi^{2}-2 j \pi \mathrm{Si}(2 \pi j)\right]
$$

Proof. For part (a) we use the combination

$$
\{x\}^{2}=P_{2}(x)+P_{1}(x)+\frac{1}{3}=\sum_{j=1}^{\infty}\left[\frac{\cos (2 \pi j x)}{\pi^{2} j^{2}}-\frac{\sin (2 \pi j x)}{\pi j}\right]+\frac{1}{3},
$$


and carry out the integrations. For part (b) we again use the Fourier series (2.35), so that

$$
\{x\}^{3}=P_{3}(x)+\frac{3}{2} P_{2}(x)+P_{1}(x)+\frac{1}{4}=\sum_{j=1}^{\infty}\left[\frac{3}{2} \frac{\cos (2 \pi j x)}{\pi^{2} j^{2}}+\left(\frac{3}{2 \pi^{2} j^{2}}-1\right) \frac{\sin (2 \pi j x)}{\pi j}\right]+\frac{1}{4} .
$$

Proposition 3. From (e.g., [11], p. 107)

$$
\ln \Gamma(a)=\left(a-\frac{1}{2}\right) \ln a-a+\frac{1}{2} \ln (2 \pi)-\int_{0}^{\infty} \frac{P_{1}(t)}{t+a} d t
$$

we have

$$
\psi(a)=\ln a-\frac{1}{2 a}+\int_{0}^{\infty} \frac{P_{1}(t)}{(t+a)^{2}} d t .
$$

Using the Fourier representation (2.29) and performing the integration gives the Proposition.

Remarks. When $a=1$ in (1.21) we recover (2.27), while from the case $a=1 / 2$ we find the companion relation

$$
\gamma+\ln 2=1-2 \sum_{j=1}^{\infty}(-1)^{j} \operatorname{Ci}(\pi j)
$$

The sum on the right side of this equation may be determined similarly to how we found (2.31).

Similar series representations in terms of $\mathrm{Si}$ and $\mathrm{Ci}$ may be obtained for $\ln \Gamma(a)$ and for the polygamma functions $\psi^{(j)}(a)$. For instance, from $(2.39)$ we have

$$
\begin{gathered}
\ln \Gamma(a)=\left(a-\frac{1}{2}\right) \ln a-a+\frac{1}{2} \ln (2 \pi) \\
+\frac{1}{2 \pi} \sum_{j=1}^{\infty} \frac{1}{j}[2 \sin (2 \pi j a) \operatorname{Ci}(2 \pi j a)+\cos (2 \pi j a)[\pi-2 \operatorname{Si}(2 \pi j a)]] .
\end{gathered}
$$


From this equation at $a=1$ we may conclude that

$$
\frac{1}{2 \pi} \sum_{j=1}^{\infty} \frac{1}{j}[\pi-2 \operatorname{Si}(2 \pi j)]=1-\frac{1}{2} \ln (2 \pi),
$$

and at $a=1 / 4$ that

$\ln \Gamma\left(\frac{1}{4}\right)=\frac{1}{2} \ln (4 \pi)-\frac{1}{4}+\frac{1}{2 \pi}\left\{\frac{1}{2} \sum_{m=1}^{\infty} \frac{(-1)^{m}}{m}[\pi-\operatorname{Si}(\pi m)]+2 \sum_{m=0}^{\infty} \frac{(-1)^{m}}{(2 m+1)} \operatorname{Ci}[\pi(m+1 / 2)]\right\}$.

We give a second direct proof of relation (2.43). We have

$$
\begin{gathered}
\sum_{j=1}^{\infty} \frac{1}{j}[\pi-2 \operatorname{Si}(2 \pi j)]=2 \sum_{j=1}^{\infty} \frac{1}{j}[\operatorname{Si}(\infty)-\operatorname{Si}(2 \pi j)]=2 \sum_{j=1}^{\infty} \frac{1}{j} \int_{2 \pi j}^{\infty} \frac{\sin t}{t} d t \\
=2 \sum_{j=1}^{\infty} \frac{1}{j} \int_{0}^{\infty} \frac{\sin v}{v+2 \pi j} d v=2 \sum_{j=1}^{\infty} \frac{1}{j} \int_{0}^{\infty} \sin v d v \int_{0}^{\infty} e^{-(v+2 \pi j) x} d x \\
=-2 \int_{0}^{\infty} \sin v d v \int_{0}^{\infty} e^{-x v} \ln \left(1-e^{-2 \pi x}\right) d x=-2 \int_{0}^{\infty} \frac{\ln \left(1-e^{-2 \pi x}\right)}{1+x^{2}} d x \\
=4 \pi \int_{0}^{\infty} \frac{\tan ^{-1} x}{e^{2 \pi x}-1} d x .
\end{gathered}
$$

In the last step we integrated by parts. We now differentiate a known result [19] (p. 100), to write

$$
\begin{aligned}
\zeta^{\prime}(s)=- & \frac{1}{(s-1)^{2}}+2 \int_{0}^{\infty} \frac{\cos \left(s \tan ^{-1} t\right)}{\left(1+t^{2}\right)^{s / 2}} \frac{\tan ^{-1} t}{\left(e^{2 \pi t}-1\right)} d t \\
& -\int_{0}^{\infty} \frac{\sin \left(s \tan ^{-1} t\right)}{\left(1+t^{2}\right)^{s / 2}} \frac{\ln \left(1+t^{2}\right)}{\left(e^{2 \pi t}-1\right)} d t .
\end{aligned}
$$

Since by (2.46) we have

$$
\zeta^{\prime}(0)=-\frac{1}{2} \ln (2 \pi)=-1+2 \int_{0}^{\infty} \frac{\tan ^{-1} t}{e^{2 \pi t}-1} d t
$$


the result (2.43) again follows.

Proposition 4. We first demonstrate part (b), with part (a) being similar. We use the representation for $\operatorname{Re} x>0$ (e.g., [12], p. 928)

$$
\operatorname{Ci}(x)=\gamma+\ln x+\int_{0}^{x} \frac{(\cos t-1)}{t} d t
$$

We also use the Fourier expansions

$$
\sum_{n=1}^{\infty} \frac{\cos (\beta n)}{n^{4}}=-\frac{\beta^{4}}{48}+\frac{\pi}{12} \beta^{3}-\frac{\pi^{2}}{12} \beta^{2}+\zeta(4)
$$

and

$$
\sum_{n=1}^{\infty}(-1)^{n} \frac{\cos (\beta n)}{n^{4}}=-\frac{\beta^{4}}{48}+\frac{\pi^{2}}{24} \beta^{2}-\frac{7}{8} \zeta(4)
$$

Then we have

$$
\begin{gathered}
\sum_{n=1}^{\infty} \frac{\operatorname{Ci}(\beta n)}{n^{4}}=\sum_{n=1}^{\infty} \frac{1}{n^{4}}\left[\gamma+\ln (\beta n)+\int_{0}^{\beta n} \frac{(\cos t-1)}{t} d t\right] \\
=(\gamma+\ln \beta) \zeta(4)-\zeta^{\prime}(4)+\sum_{n=1}^{\infty} \int_{0}^{1} \frac{[\cos (\beta n v)-1]}{v} d v \\
=(\gamma+\ln \beta) \zeta(4)-\zeta^{\prime}(4)+\frac{\beta^{2}}{12} \int_{0}^{1}\left(-\frac{\beta^{2}}{4} v^{3}+\pi \beta v^{2}-\pi^{2} v\right) d v \\
=(\gamma+\ln \beta) \zeta(4)-\zeta^{\prime}(4)+\frac{\beta^{2}}{12}\left(-\frac{\beta^{2}}{16}+\frac{\pi}{3} \beta-\frac{\pi^{2}}{2}\right) .
\end{gathered}
$$

The integral over $v$ being absolutely convergent, the interchange of summation and integration is justified. For $(1.22 \mathrm{~d})$, we use the alternating zeta function for $\operatorname{Re} s>0$ ([12], p. 1073, or [19], p. 96)

$$
\sum_{n=1}^{\infty} \frac{(-1)^{n}}{n^{s}}=\left(2^{1-s}-1\right) \zeta(s)
$$


so that

$$
\sum_{n=1}^{\infty} \frac{(-1)^{n}}{n^{s}} \ln n=\left(1-2^{1-s}\right) \zeta^{\prime}(s)+2^{1-s}(\ln 2) \zeta(s) .
$$

For part (c) we use the summations [14] (p. 244)

$$
\sum_{n=1}^{\infty} \frac{\cos n x}{n^{2 k}}=\frac{(-1)^{k-1}}{2(2 k) !}(2 \pi)^{2 k} B_{2 k}\left(\frac{x}{2 \pi}\right),
$$

and

$$
\sum_{n=1}^{\infty}(-1)^{n} \frac{\cos n x}{n^{2 k}}=\frac{(-1)^{k-1}}{2(2 k) !}(2 \pi)^{2 k} B_{2 k}\left(\frac{x+\pi}{2 \pi}\right)
$$

For part (d) we use the $y=0$ case of the two summations [14 (p. 244)

$$
\begin{aligned}
\sum_{k=1}^{\infty} \frac{\cos (k x+y)}{k^{a}}= & \frac{(2 \pi)^{a}}{2 \Gamma(a)} \csc (\pi a)\left[\sin \left(y+\frac{\pi a}{2}\right) \zeta\left(1-a, 1-\frac{x}{2 \pi}\right)\right. \\
& \left.-\sin \left(y-\frac{\pi a}{2}\right) \zeta\left(1-a, \frac{x}{2 \pi}\right)\right]
\end{aligned}
$$

and

$$
\begin{gathered}
\sum_{k=1}^{\infty}(-1)^{k} \frac{\cos (k x+y)}{k^{a}}=\frac{(2 \pi)^{a}}{2 \Gamma(a)} \csc (\pi a)\left[\sin \left(y+\frac{\pi a}{2}\right) \zeta\left(1-a, \frac{\pi-x}{2 \pi}\right)\right. \\
\left.-\sin \left(y-\frac{\pi a}{2}\right) \zeta\left(1-a, \frac{\pi+x}{2 \pi}\right)\right] .
\end{gathered}
$$

Part (e) uses

$$
\sum_{n=1}^{\infty} \frac{1}{(2 n+1)^{a}}=\left(1-2^{-a}\right) \zeta(a)-1
$$

and

$$
\sum_{n=1}^{\infty} \frac{(-1)^{n}}{(2 n+1)^{a}}=4^{-a}\left[\zeta\left(a, \frac{1}{4}\right)-\zeta\left(a, \frac{3}{4}\right)\right]-1,
$$

together with the $y=0$ case of two summations [14] (p. 245), so that

$\sum_{k=0}^{\infty} \frac{\cos k x}{(2 k+1)^{a}}=\frac{(2 \pi)^{a}}{4 \Gamma(a)} \csc (\pi a)\left\{-\sin \left(\frac{x+\pi a}{2}\right)\left[\zeta\left(1-a, \frac{x+2 \pi}{4 \pi}\right)-\zeta\left(1-a, \frac{x}{4 \pi}\right)\right]\right.$ 


$$
\left.+\sin \left(\frac{x-\pi a}{2}\right)\left[\zeta\left(1-a, \frac{2 \pi-x}{4 \pi}\right)-\zeta\left(1-a, 1-\frac{x}{4 \pi}\right)\right]\right\}
$$

and

$$
\begin{gathered}
\sum_{k=0}^{\infty}(-1)^{k} \frac{\cos k x}{(2 k+1)^{a}}=\frac{(2 \pi)^{a}}{4 \Gamma(a)} \csc (\pi a)\left\{\cos \left(\frac{x+\pi a}{2}\right)\left[\zeta\left(1-a, \frac{x+\pi}{4 \pi}\right)-\zeta\left(1-a, \frac{x+3 \pi}{4 \pi}\right)\right]\right. \\
\left.+\cos \left(\frac{x-\pi a}{2}\right)\left[\zeta\left(1-a, \frac{\pi-x}{4 \pi}\right)-\zeta\left(1-a, \frac{3 \pi-x}{4 \pi}\right)\right]\right\} .
\end{gathered}
$$

Part (f) uses [14] (p. 247)

$$
\sum_{n=1}^{\infty} \frac{z^{n} \cos n x}{n(n+1)}=1-\frac{1}{2 z}(z-\cos x) \ln \left(1-2 z \cos x+z^{2}\right)-\frac{\sin x}{z} \tan ^{-1}\left(\frac{z \sin x}{1-z \cos x}\right) .
$$

Remarks. In part (a), due to the functional equation of the Riemann zeta function, we may write the value $\zeta^{\prime}(2)=\zeta(2)[\gamma+\ln (2 \pi)+12 \ln A]$, where $A \simeq 1.28243$ is the Glaisher constant, such that $\ln A=1 / 12-\zeta^{\prime}(-1)=-\left[\zeta(-1)+\zeta^{\prime}(-1)\right]$.

As shown by part (c), the Proposition extends to all summations $\sum_{n=1}^{\infty}( \pm 1)^{n} \mathrm{Ci}(\beta n) / n^{2 k}$ with $k \geq 1$. Alternatively, successive Fourier expansions may be built up by twice integrating the previous one, and requiring the appropriate $\zeta(2 k)$ constant term. Of course in this part, the relation ([11], p. 14 or [12], p. 1077 or [19] p. 98) $\zeta(2 k)=(-1)^{k-1}(2 \pi)^{2 k} B_{2 k} /[2(2 k) !]$ applies.

Analogous results can be given for sums $\sum_{n=1}^{\infty}[\pi-2 \operatorname{Si}(\beta n)] / n^{2 k+1}$ with $k \geq 0$.

Part (d) of the Proposition subsumes part (c), owing to the reduction $\zeta(1-n, q)=$ $-B_{n}(q) / n$ (e.g., [19], p. 85) and the functional equation $B_{n}(1-x)=(-1)^{n} B_{n}(x)$. The latter relation shows the reflection property $B_{2 k}(1-x)=B_{2 k}(x)$.

Other $\mathrm{Ci}$ summations are possible, based for instance upon known sums for $\sum_{k=0}^{\infty}( \pm 1)^{k} a^{k} \cos k x /(k m+n)$ [14] (pp. 246-247). 


\section{Summary}

We have developed a variety of series and integral representations for a family of functions. From the representations for the polygamma functions follow many special cases, including representations of the values $\psi(p / q)$, of harmonic numbers $H_{n}$, and

of generalized harmonic numbers $H_{n}^{(r)}$. We have found representations for $\ln \Gamma$ and in turn a product representation for the Gamma function. Most of the representations are quite suited for computation, and in fact their convergence may also be accelerated. The approach via the zeroth Stieltjes constant enables the development of new representations, as well as the recovery of known series and integral representations for constants such as $\gamma$ and for the digamma function.

We have given series representations in terms of trigonometric integrals $\mathrm{Ci}$ and Si for $\gamma$, for certain combinations of $\gamma$ and $\zeta(n) / n$, for $\gamma+\ln 2$, and generally for $\ln \Gamma(a)$ and $\psi(a)$. Additionally, we have demonstrated in several ways how to perform summations over parameterized values of $\mathrm{Si}$ and $\mathrm{Ci}$. 


\section{References}

[1] M. Abramowitz and I. A. Stegun, Handbook of Mathematical Functions, Washington, National Bureau of Standards (1964).

[2] G. E. Andrews, R. Askey, and R. Roy, Special Functions, Cambridge University Press (1999).

[3] B. C. Berndt, On the Hurwitz zeta function, Rocky Mtn. J. Math. 2, 151-157 (1972).

[4] B. C. Berndt and A. Dixit, A transformation formula involving the Gamma and Riemann zeta functions in Ramanujan's lost notebook, A. Ramakrishnan memorial volume, Springer (to appear); arXiv:0904.1053v2 (2009).

[5] M. W. Coffey Addison-type series representation for the Stieltjes constants, J. Number Th. 130, 2049-2064 (2010); arXiv:0912.2391 (2009).

[6] M. W. Coffey, New results on the Stieltjes constants: Asymptotic and exact evaluation, J. Math. Anal. Appl. 317, 603-612 (2006); arXiv:math-ph/0506061.

[7] M. W. Coffey, Series representations for the Stieltjes constants, arXiv:0905.1111 (2009).

[8] M. W. Coffey, On representations and differences of Stieltjes coefficients, and other relations, to appear in Rocky Mtn. J. Math.; arXiv/math-ph/0809.3277v2 (2008). 
[9] M. W. Coffey, unpublished (2009).

[10] M. W. Coffey, On one dimensional digamma and polygamma series related to the evaluation of Feynman diagrams, J. Comput. Appl. Math. 183, 84-100 (2005).

[11] H. M. Edwards, Riemann's Zeta Function, Academic Press, New York (1974).

[12] I. S. Gradshteyn and I. M. Ryzhik, Table of Integrals, Series, and Products, Academic Press, New York (1980).

[13] R. L. Graham, D. E. Knuth, and O. Patashnik, Concrete Mathematics, AddisonWesley (1994).

[14] E. R. Hansen, A table of series and products, Prentice-Hall (1975).

[15] A. Ivić, The Riemann Zeta-Function, Wiley New York (1985).

[16] C. Knessl and M. W. Coffey, An asymptotic expression for a sum $S_{\gamma}(n)$ appearing under the Li criterion, preprint (2010).

[17] B. Riemann, Über die Anzahl der Primzahlen unter einer gegebenen Grösse, Monats. Preuss. Akad. Wiss., 671 (1859-1860).

[18] J. Sondow, An infinite product for $e^{\gamma}$ via hypergeometric formulas for Euler's constant $\gamma$, arXiv:math/0306008v1 (2003).

[19] H. M. Srivastava and J. Choi, Series associated with the zeta and related functions, Kluwer (2001). 
[20] T. J. Stieltjes, Correspondance d'Hermite et de Stieltjes, Volumes 1 and 2, Gauthier-Villars, Paris (1905).

[21] E. C. Titchmarsh, The Theory of the Riemann Zeta-Function, 2nd ed., Oxford University Press, Oxford (1986).

[22] J. R. Wilton, A note on the coefficients in the expansion of $\zeta(s, x)$ in powers of s - 1, Quart. J. Pure Appl. Math. 50, 329-332 (1927). 